\title{
Positive Shifts in Emotion Evaluation Following Mindfulness-Based Cognitive Therapy (MBCT) in Remitted Depressed Participants
}

\author{
Kate Williams $^{1,2}$ (D) $\cdot$ Rebecca Elliott $^{1} \cdot$ Thorsten Barnhofer $^{3} \cdot$ Roland Zahn $^{4} \cdot$ lan M. Anderson ${ }^{1}$
}

Accepted: 10 October 2020 / Published online: 5 November 2020

(C) The Author(s) 2020

\begin{abstract}
Objectives A combination of negatively biased information processing and a reduced ability to experience positive emotions can persist into remission from major depression (rMDD). Studies have shown that mindfulness-based cognitive therapy (MBCT) can increase self-reported positive emotions in rMDD participants; similar changes using neuropsychological tasks have not been shown. In this study, we investigated neuropsychological change in emotional processing following MBCT in rMDD participants.

Methods Seventy-three rMDD participants, 40 of whom received MBCT and 33 of whom continued with treatment as usual (TAU), and 42 never depressed participants took part; neither the TAU nor never depressed participants received MBCT. All were assessed at baseline and immediately following MBCT or after an 8-week gap for those without active intervention. Participants completed emotion evaluation and face emotion recognition tasks with self-report measures (mood, mindfulness) at each session.

Results Results showed an MBCT-specific shift in ratings from less negative to more positive emotion evaluations, which correlated with mindfulness practice and self-report mindfulness change. Both the MBCT and TAU groups showed a small increase in overall face emotion recognition accuracy compared with no change in never depressed participants.

Conclusions These findings support a specific role for MBCT in encouraging more positive evaluations of life situations in those with previous depression rather than influencing lower-level processing of emotions. Results should be interpreted cautiously given that this was a non-randomised, preference choice trial.
\end{abstract}

Trial Registration NCT02226042

Keywords Mindfulness-based cognitive therapy $\cdot$ MBCT $\cdot$ Mindfulness $\cdot$ Remitted major depression $\cdot$ Emotion processing · Positive emotions

Electronic supplementary material The online version of this article (https://doi.org/10.1007/s12671-020-01521-4) contains supplementary material, which is available to authorized users.

Kate Williams

kate.williams-4@manchester.ac.uk

1 Neuroscience and Psychiatry Unit, University of Manchester, Manchester M13 9PT, UK

2 Present address: Division of Clinical Psychology, University of Manchester, 2nd Floor Zochonis Building, Brunswick Street, Manchester M13 9PL, UK

3 Department of Psychology, University of Surrey, Guildford GU2 7XH, UK

4 Centre for Affective Disorders, King's College London, London SE5 8AF, UK
The cognitive model underlying depression outlines how the experience of early adverse events can lead to the internalisation of negative self-referential thoughts, leading to the development of "latent schemas" (Beck 2008). Such schemas may then be reactivated by either internal or external events whereby repeated reactivation of these schemas over time influences the increased acquisition and processing of negative information (Beck 1967). Such negatively biased information processing may start to influence appraisals and interpretations, thus increasing vulnerability to develop or maintain depression (Disner et al. 2011). Further, even when in remission from depression, self-negative thinking patterns that have become associated with low mood when depressed may become more easily activated by internal or external events (Scher et al. 2005). This reactivation of negative thinking during remission has been linked with vulnerability to relapse into depression (Segal et al. 2006). 
Alongside this increase in negatively biased information processing, people who are currently depressed may encounter a reduced ability to experience positive emotions (Pizzagalli et al. 2008). Indeed, studies have shown that people experiencing depression may show a blunted response to positive cues, a lack of a positivity bias, and diminished reward responsiveness (Henriques and Davidson 2000; McCabe and Gotlib 1995; Pizzagalli et al. 2005). Further, this reduced ability to experience positive emotions can persist into remission (Pechtel et al. 2013; Weinberg and Shankman 2017). The combination of negatively biased information processing and diminished ability to experience positive emotions may contribute to negative downward spirals of mood and negative thinking during remission, potentially leading to relapse into depression (Garland et al. 2010).

Mindfulness-based cognitive therapy (MBCT) was developed as a relapse-prevention intervention for people in remission from major depression (rMDD; Segal et al. 2013). The mindfulness-based practices taught throughout MBCT encourage a recognition of and disengagement from negative thinking patterns, aiming to reduce the negative information processing biases. Simultaneously, mindfulness-based practices encourage recognition and acceptance of all experience; be it pleasant, negative, or neutral. By encouraging an open, non-judgemental approach towards all emotions and thoughts, participants of MBCT are taught to take a broader perspective on their emotional experience. Consistent with this, studies have shown that MBCT reduces negative information processing biases including reducing rumination and increasing the ability to decentre from negative thought patterns (Hargus et al. 2010; Michalak et al. 2011; Shahar et al. 2010; Teasdale et al. 2002; van Aalderen et al. 2012; van Den Hurk et al. 2012). There is also initial evidence that MBCT increases the experiencing of positive emotions alongside reducing residual depression symptoms (Garland et al. 2015; Geschwind et al. 2011). However, to date, the evidence for the effect of $\mathrm{MBCT}$ on modifying emotional processing biases has largely come from self-report questionnaires, which can be influenced by participant expectations or hopes for change, and also lack a realistic, everyday, context which limits interpretation of their real-life impact and of any changes seen. Nonquestionnaire measures completed within the context of images of everyday scenarios potentially provide an opportunity for assessments more relevant to actual experience.

More specifically, neuropsychological investigations of emotional processing have included studies on face emotion recognition biases, reflecting early, automatic processing, which have suggested processing biases towards negative and away from positive faces in depression (Bourke et al. 2010; Leppänen 2006; Shiroma et al. 2016; Weightman et al. 2014). This may persist into remission and contribute to relapse vulnerability, although the evidence is somewhat conflicting (Anderson et al. 2011; Joormann and Gotlib
2007; LeMoult et al. 2009; Münkler et al. 2015). Further, considering higher level emotional processing, impairments across a range of social aspects including social communication, perception, and increased sensitivity to social rejection have been reported in depression, with some evidence suggesting that impairments can persist into remission (Kupferberg et al. 2016; Rhebergen et al. 2010).

Therefore, given the evidence that impairments can persist into remission and the lack of experimental measures to capture changes in emotional processing following MBCT, we selected two tasks to investigate both lower level face emotion recognition and higher level emotion evaluations across a range of social or autonomous situations. We aimed to investigate whether MBCT can modify the identification and experience of different emotions across a range of situations using experimental tasks before and after MBCT. To control for non-specific and practice effects, we included two control groups: rMDD participants who did not receive MBCT (treatment as usual; TAU) and never depressed participants (healthy volunteers, HVs). At baseline, we expected both rMDD groups to show an enhanced bias towards recognising or evaluating negative emotions and an enhanced bias away from recognising or evaluating positive emotions on both tasks, compared to HVs. Following MBCT, we hypothesised that MBCT participants would show an increased facilitation towards positive stimuli (i.e. increased positive emotion evaluations and recognition of positive faces) and decreased facilitation towards negative stimuli (i.e. reduced bias towards recognising and evaluating negative emotions) compared with both rMDD control (TAU) and HV groups. Finally, we hypothesised that changes would correlate with both engagement with mindfulness practice during $\mathrm{MBCT}$ and self-report mindfulness change.

\section{Method}

\section{Participants}

Full details on recruitment numbers are listed in Fig. 1. rMDD $(N=124)$ and never depressed, healthy volunteers (HVs; $N=$ 46) were screened. rMDD participants were recruited from a departmental database, posters at local general practice and community centres, and both rMDD and HV groups were recruited from posters around the university campus, online university research announcements, community websites, and social media. Advertisements for rMDD participants specifically outlined that participants could take part in MBCT or join the comparison control group (TAU), according to preference or practicality. Final numbers included $40 \mathrm{MBCT}$ and 33 TAU (both rMDD) participants, and 42 HVs. Ten TAU participants were originally recruited to the MBCT condition (see the "Design" section below). The planned sample size of 
Fig. 1 CONSORT flowchart for recruitment of all participants. Note. DNA = did not attend; HVs = healthy, never depressed, volunteers; rMDD = remitted major depressive disorder; TAU = treatment as usual; out of 11 participants who dropped out after three or fewer MBCT sessions, one did not reply to our invitation to join the TAU group and we lost all contact with them; only 10 continued into TAU. Although one participant expressed that their reason for dropping out was due to MBCT increasing their anxiety, they did not meet DSM-IV criteria at that point (or at post-session) for an MDD relapse

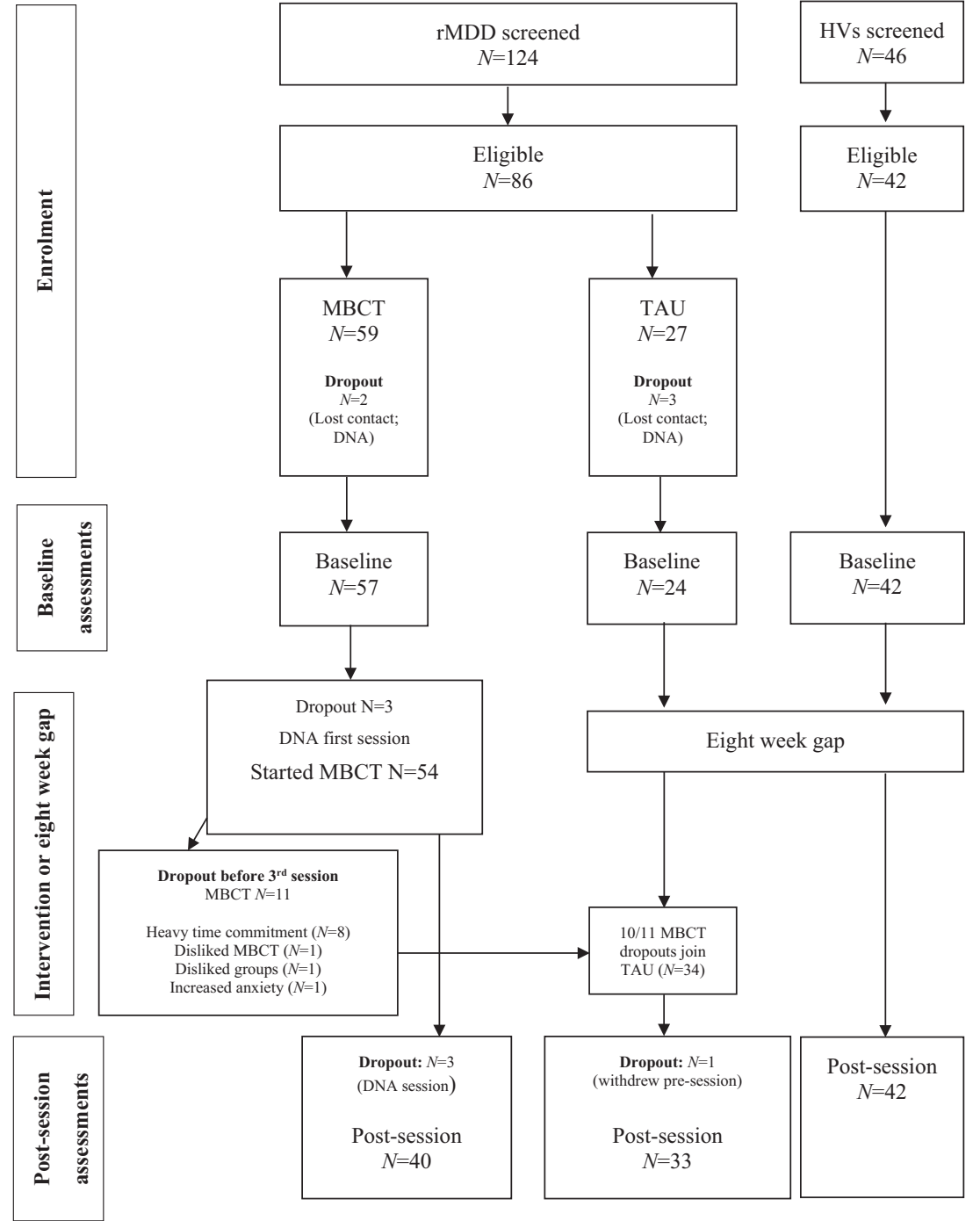

40 participants per group gave $80 \%$ power to detect an effect size of .65 at $p=.05$. Ethical approval was granted by the NHS (North West - Preston Research Ethics Committee) and participants gave written informed consent in line with the Declaration of Helsinki (World Medical Association 2001).

Inclusion and Exclusion Criteria Participants had experienced at least two major depressive (MDD) episodes in the last 5 years and had been in full or partial remission according to DSM-IV criteria for the last 3 months as assessed with the Mini International Neuropsychiatric Interview (MINI; Sheehan et al. 1998) with a cut off score of $\leq 12$ on the MADRS (Montgomery-Åsberg Depression Rating Scale; Montgomery and Asberg 1979). rMDD participants taking antidepressants were recruited if there were no recent (within the last 3 months) or planned future changes (during the study) to either dosage or medication. This was monitored throughout the study to ensure that there were no additions or changes to pre-existing treatments for those taking part. All participants were aged 18 to 60 , in good physical health, had normal colour vision, and were sufficiently fluent in English to ensure task understanding. Groups were closely matched for age, gender, and estimated IQ (see the "Measures" section below). Participants were excluded if they had any other current or previous DSM-IV axis 1 mental health diagnosis (with the exception of anxiety disorders provided this was secondary to a diagnosis of remitted major depression), substance abuse or dependence, or self-reported physical or neurological disorders; HVs were excluded if they had any current or previous DSM-IV axis 1 mental health diagnosis (including MDD) assessed using the MINI (Sheehan et al. 1998). Any participants with previous participation in a mindfulness-based intervention (MBI), a regular mindfulness practice, or any ongoing or completion of psychotherapy in the last 12 months were excluded, and both control groups were asked not to take up any mindfulness practice during the study. 


\section{Procedure}

Mood and estimated IQ measures were completed at screening. rMDD participants provided a timeline of lifetime MDD episodes, giving details from their most recent two episodes (duration, symptoms, severity, and treatments) to ensure both met DSM-IV criteria for MDD. All participants completed questionnaires and neuropsychological tasks at baseline and 8 weeks later (either after MBCT or an 8-week gap). The MADRS and the retrospective measure of amount of mindfulness practice were completed at the beginning of sessions, and all other questionnaires completed at the end of each session.

\section{Design}

This study was a prospective, non-randomised preference choice mechanistic design and is registered on and includes details regarding the planned data analyses on clinicaltrials.gov (NCT02226042). This study includes a subset of the planned analyses as proposed in the study protocol and primary trial outcomes have been reported elsewhere (Williams 2018; Williams et al. 2020; Williams et al., in preparation). MBCT participants received the 8-week MBCT treatment free-ofcharge. TAU and HV participants were recruited as controls for change over time, with 8 weeks between baseline and post-assessments. Control groups did not receive MBCT but received a small financial reimbursement plus travel expenses. As the study was designed to study the mechanisms involved in MBCT, participants who dropped out of MBCT after three or fewer sessions (i.e. having limited practice with mindfulness) were invited to continue in the study and were analysed with the TAU group $(N=10$; see the "Data Analyses" section and Fig. 1).

Intervention MBCT was delivered according to the manual (Segal et al. 2013) and in adherence with the UK Network for Mindfulness-based Teachers Good Practice Guidelines (http://www.mindfulnessteachersuk.org.uk/) to a total of five groups between 2015 and 2017, each with between 7 and 15 participants. MBCT involved weekly 2 -h sessions over 8 weeks with an all-day mindfulness retreat day around week 6. Participants were given CDs and online access to audio practices and invited to practice in-between sessions. All MBCT groups were delivered by two mindfulness-based teachers (KW and an external teacher) who have both undergone recognised mindfulness teacher training with accredited organisations belonging to the above network. Both had a minimum of 7 years of personal mindfulness practice and continuing engagement with training.

\section{Measures}

Mini International Neuropsychiatric Interview (Sheehan et al. 1998) The MINI is a structured experimenter-rated interview for diagnosis of axis-1 psychiatric disorders of the DSM-IV and ICD-10 classification systems, with high inter-rater reliability of above .75 for all diagnoses. The MINI was conducted by the lead author who was trained in its administration.

Montgomery-Åsberg Depression Rating Scale The MADRS is a widely used measure of depression severity. It includes 10 experimenter-rated items of depression symptoms on a scale of 0 (absent/occasional) to 6 (severe/continuous) over the last week. Internal consistency in this sample was $\alpha=.78$ at baseline and $\alpha=.87$ post-MBCT.

Estimated Verbal IQ: Wechsler Test of Adult Reading (Psychological Corporation 2001) Participants read aloud 50 commonly misspelled words. The number of correctly pronounced words was converted to a standard score using age, gender, and education level, to predict verbal IQ, to limit any potential confounds from IQ between groups with regard to performance across neuropsychological measures. The scale has high internal consistency between .87 and .95 .

Five Facet Mindfulness Questionnaire (Baer et al. 2006) The Five Facet Mindfulness Questionnaire (FFMQ) includes 39 items asking participants to rate on a scale of 1 (never or rarely true) to 5 (very often or always true) how often they engage in behaviours or thoughts (e.g. "I find myself doing things without paying attention"). Previous studies have identified a poor fit of the five-factor model to both community and clinical samples (Gu et al. 2015; Williams et al. 2014) and recommend removing "observing"; thus, we have analysed the FFMQ-4 factor model (31 items) only (reflecting the subscales "describing", "acting with awareness", "non-judging of inner experience", "nonreactivity to inner experience"). Internal consistency in this sample was $\alpha=.92$ at baseline and $\alpha=.92$ post-MBCT.

Retrospective Amount of Mindfulness Practice At the start of each MBCT session, participants completed a questionnaire about which formal mindfulness practices they had used (e.g. mindful breathing) and the duration of each that they completed on each day of the previous week.

Neuropsychological Tasks The neuropsychological tasks were selected to investigate higher and lower level emotional processing based on evidence of a persistence of impairments in social emotion processing and deficits in face emotion recognition on remission from depression (Anderson et al. 2011; Elliott et al. 2012; Saris et al. 2017). Test-retest reliability was calculated for both tasks with results showing moderate to 
excellent reliability between baseline and post-session variables (see Supplementary Material). Tasks were piloted beforehand and were programmed using E-Prime Professional 2 (Psychology Software Tools 2012).

Emotion Evaluation Task (Elliott et al. 2012) This task was designed to probe a continuum of positive and negative feelings in response to images of social and autonomous situations depicting success and failure, as well as neutral (nonemotive) scenarios. There were 16 social ( 8 positive (inclusion), 8 negative (exclusion)), 16 autonomous ( 8 positive (success), 8 negative (failure)), and eight neutral scenarios (4 social, 4 autonomous), each including one person highlighted by a red circle. Using numbers on the keyboard, participants rated how they would feel if they were the highlighted person in the scenario from 1 (extremely sad) to 7 (extremely happy). Stimuli were randomly (non-repeating) presented and the task was self-paced with each subsequent image appearing on the screen once the participant submitted their rating. Participants were encouraged to respond with their first instinct. Figure 2 shows examples of different types of scenarios.

Face Emotion Recognition Task This task (Anderson et al. 2011) was designed to probe the ability to recognise a range of facial emotions using standardised morphed faces (Ekman and Friesen 1976) depicting the six universally recognised emotions as defined by Ekman (1992): happiness, anger, sadness, surprise, fear, and disgust. A neutral face was included as a control for affect. Each emotional face was morphed with neutral to give three different intensities: 30,50 , and $70 \%$. Morphing allows for either analysis of total intensities (summed across all three intensities) or investigation of different intensities; however, analysing each intensity separately would limit the power as only four faces per emotion were displayed at each of the three intensities. Participants were asked to identify the emotion of each face (anger, disgust, fear, happy, sad, surprise, neutral) using labelled keys on the keyboard. There were 84 faces in total with 12 per emotion. Each face was presented individually for $500 \mathrm{~ms}$ (modified from a 1-s presentation in Anderson et al. 2011) followed by a fixation cross of $4500 \mathrm{~ms}$. The task lasted 6 min including three practice blocks. Figure 3 shows examples of each face and emotion.

\section{Data Analyses}

All data were analysed using SPSS v23. The primary analysis was a per protocol (PP) analysis given the mechanistic aims of the study (see the "Design" section). For the emotion evaluation task, the primary outcome was the overall, or composite, score (mean score across all emotional conditions) with individual conditions examined as an exploratory analysis by including them as a within-subject factor. For the face emotion recognition task, we analysed total hit proportions (accuracy) for each emotion (all three intensities combined with 12 trials per emotion) and total false alarm proportions (out of 72 remaining trials). Composite accuracy and false alarm

Fig. 2 Emotion evaluation task examples of hypothetical scenarios. Note. Scenarios depict the following conditions: social exclusion, autonomous success, and neutral social. Of note, in all our analyses, we have reversed the direction of the ratings so that lower ratings represent negative scores (e.g. 1 = "extremely sad") and higher ratings represent positive scores (e.g. 7 = "extremely happy")

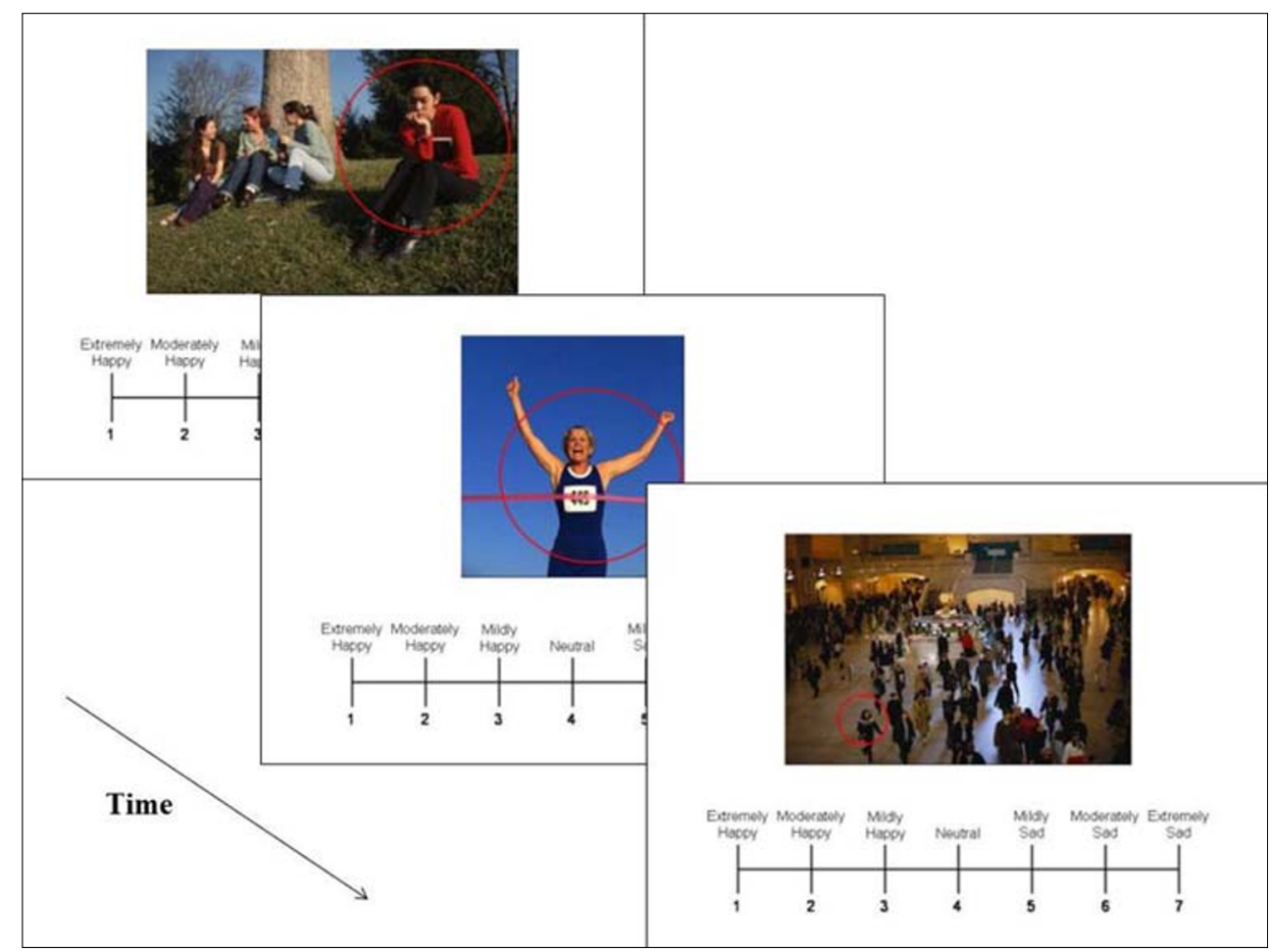


Fig. 3 Face emotion processing task. Examples of faces
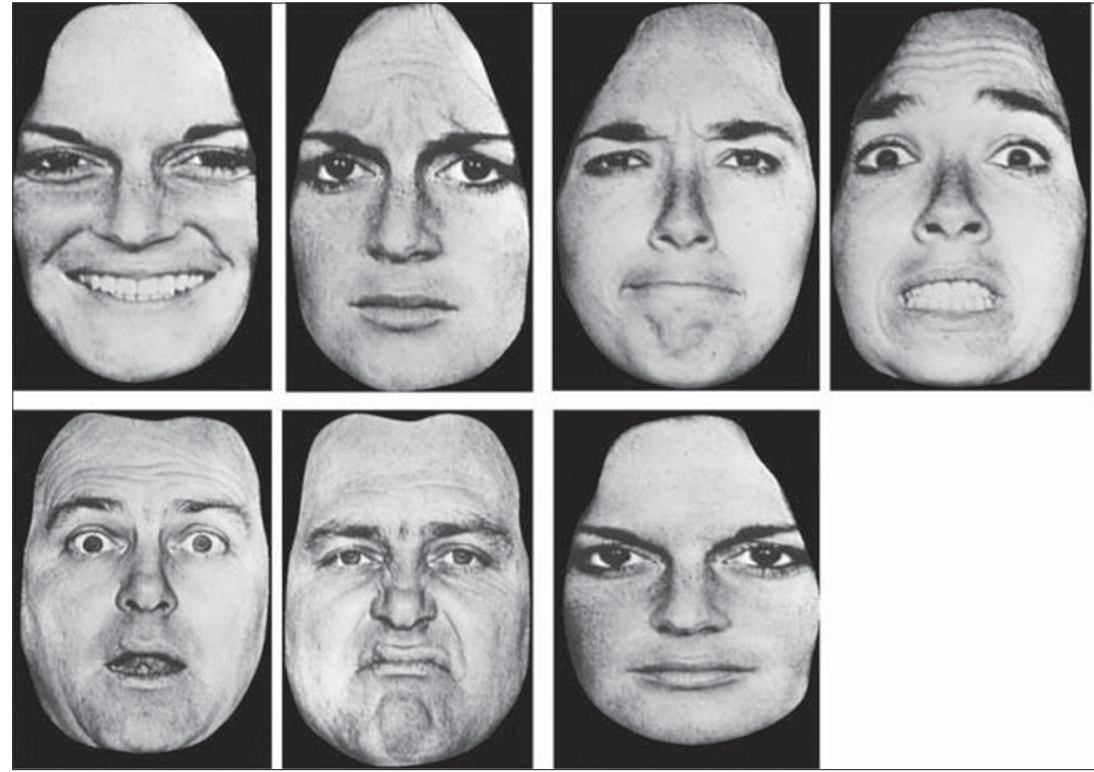

proportions for all emotions were also calculated as a general measure of accuracy in recognising facial emotions. Reaction times (RTs) were not analysed as participants were not asked to respond as quickly as possible.

Both tasks were analysed using repeated measures ANOVAs (rmANOVAs) on change scores (post-baseline) as our expectation was that the rMDD participants would differ from HVs at baseline (van Breukelen 2013). Valence was the within-subject factor (e.g. emotion condition, individual face emotions) and group as the between-subjects factor (MBCT, TAU, HV). Corrected statistics (HuynhFeldt) were applied when the normality assumption was violated (written as $H F \mathrm{~F}$ ) with uncorrected degrees of freedom values given for clarity. Baseline scores were analysed using rmANOVAs to identify potential group difference pre-intervention to aid further interpretation of change scores (post-intervention scores are also provided in Supplementary Material). For significant rmANOVA findings, Dunnett's test was used to compare the MBCT group with TAU and HV groups, and one-sample $t$ test on group change scores to identify whether a significant change over time had occurred.

We conducted three sensitivity analyses: (1) intentionto-treat (ITT) analyses with the participants in their original group assignments, (2) including only unmedicated $\operatorname{MBCT}(N=24)$ and TAU $(N=22)$ participants (to exclude possible medication effects), and (3) in order to control for potential baseline differences, an analysis of covariance (ANCOVA) on post-intervention composite scores covaried for pre-intervention scores in rMDD participants alone in both PP and ITT groupings. Each of the sensitivity analyses is reported briefly in the main text and in full detail in Supplementary Material.
In all groups, correlations were run between outcomes showing significant group changes and self-report mindfulness change scores (all post-baseline). In the MBCT group only, correlations were run between outcomes and mean minutes of mindfulness practice and days of practice per week during MBCT. Minutes of mindfulness practice data were non-normally distributed with one outlier $(M=59.3 \mathrm{~min})$; correlations were run with and without this participant with no changes to the results; thus, correlations in the full sample were reported.

\section{Results}

Groups did not significantly differ on age, gender distribution, or estimated IQ. rMDD groups did not significantly differ on baseline mood, self-reported mindfulness, medication status, previous episodes, age of onset, or months since their last episode (see Table 1). In both rMDD groups, baseline scores on the MADRS and FFMQ-4 were significantly different compared with HVs $(F(2,112)=20.3, p<.001 ; F(2,112)=$ 43.3; $p<.001$ respectively; see Table 1$)$. MBCT attendance was high with an average of $7.7(S D=1.4)$ sessions attended (out of nine, including the all-day practice). During MBCT, the average time participants engaged in daily formal (guided) practice was $19.1 \mathrm{~min}(S D=10.9)$ over a mean of $4.1(S D=$ 1.6) days per week. Post-intervention MADRS scores did not change significantly within or between groups but there was a significant difference between groups in the FFMQ-4 score change $(F(2,110)=8.4, p<.001)$ with the MBCT group showing a significant increase compared with both TAU $(p=.02)$ and HVs $(p<.001$; see Supplementary Material). 
Table 1 Demographics

\begin{tabular}{llll}
\hline & MBCT & TAU & HV \\
& $N=40$ & $N=33$ & $N=42$ \\
\hline Age & $37.6(11.4)$ & $33.4(10.9)$ & $33.3(10.1)$ \\
Gender (\% female) & 70 & 73 & 67 \\
Estimated IQ & $111.5(6.1)$ & $110.6(6.4)$ & $109.6(6.3)$ \\
Baseline mood (MADRS) & $4.8 * * *(4.2)$ & $4.2 * * *(3.6)$ & $.62(1.0)$ \\
Baseline mindfulness (FFMQ-4) & $82.5 * * *(16.6)$ & $84.1 * * *(17.6)$ & $112.8(15.2)$ \\
Antidepressant medication (\%) & 40 & 33 & - \\
Previous number of episodes & $6.2(3.7)$ & $6.6(3.7)$ & - \\
Age of onset & $20.2(11.5)$ & $17.3(6.6)$ & - \\
Months since last MDD episode & $12.9(11.1)$ & $11.8(10.3)$ & - \\
\hline
\end{tabular}

Means and standard deviations (in parentheses) are displayed; statistics: one-way ANOVAs or Chi squared; "_" = not applicable

$H V$ healthy, never depressed, volunteers; MADRS Montgomery-Åsberg Depression Rating Scale; MDD major depressive disorder

$* * * p<.001$ vs. HVs. rMDD groups did not significantly differ on IQ $(p=.58)$, the MADRS $(p=.57)$, the FFMQ$4(p=.70)$, previous number of episodes $(p=.69)$, age of onset $(p=.20)$, months since last MDD episode $(p=.65)$, or for antidepressant medication $(p=.63)$

\section{Emotion Evaluation Task}

\section{Baseline Cross-sectional Comparisons}

The rmANOVA showed a significant main effect for condition $\left(H F F(4,448)=1825.1, p<.001, n p^{2}=.94\right)$ and a trend towards a main effect for group $(F(2,112)=2.7, p=.07$, $\left.\eta \mathrm{p}^{2}=.05\right)$, but a non-significant condition by group interaction $\left(H F F(8,448)=.78, p=.57, \eta p^{2}=.01\right)$. Both positive and neutral scenarios were rated more positively than negative scenarios. rMDD groups tended to rate scenarios more negatively than HVs (Dunnett's test MBCT vs. HV $p=.06$; Table 2).

\section{Change Scores}

The rmANOVA showed a significant main effect for group $\left(F(2,112)=3.2, p=.04, n p^{2}=.06\right)$, but not for condition $(H F$ $\left.F(4,448)=.35, p=.82, \eta p^{2}=.003\right)$ or a condition by group interaction $\left(H F F(8,448)=.80, p=.58, \eta^{2}=.01\right)$. The MBCT group showed greater positive change in overall composite ratings than both TAU and HVs; post hoc comparisons (Dunnett's test) showed a significant difference between MBCT and TAU, with trend differences between MBCT and HV (MBCT vs. TAU $p=.045$ and MBCT vs. HV $p=.07$ respectively; Fig. 4a; Table 2). Only the MBCT group showed a significant change over time in a one-sample $t$ test (MBCT $p=.009$, TAU $p=.90$, HV $p=.68$ ). The MBCT group showed consistent numerical increases in ratings in each condition, with the greatest magnitude in social inclusion and exclusion conditions (Fig. 4b). Sensitivity analyses on the ITT population and those not taking medication showed the same overall pattern of results although the group effect was not statistically significant in the latter (see Supplementary Material). The ANCOVA on rMDD participants alone showed that the MBCT group had significantly more positive post-intervention scores than TAU in both the PP and ITT analyses covaried for pre-intervention values (see Supplementary Material).

\section{Face Emotion Recognition}

Due to scheduling difficulties, two MBCT group participants did not take part in this task.

\section{Baseline Cross-sectional Comparisons}

For accuracy and false alarms, the rmANOVAs showed significant main effects for emotion $(H F F(6,666)=133.9$, $\left.p<.001, \eta \mathrm{p}^{2}=.55 ; \operatorname{HF} F(6,666)=218.0, p<.001, \mathrm{np}^{2}=.66\right)$ and a non-significant difference between groups $(F(2,111)=$ $\left.1.01, p=.37, \mathrm{np}^{2}=.02 ; F(2,111)=.69, p=.51, \mathrm{np}^{2}=.01\right)$ and non-significant emotion by group interactions $(H F$ $F(12,666)=1.11, p=.35, \eta p^{2}=.02 ; H F F(12,666)=1.05$, $\left.p=.40, n p^{2}=.02\right)$. Happy and neutral were the most, and anger the least, accurately recognised, with the most false alarms for neutral and the least for happy (Table 2).

\section{Change Scores}

For accuracy and false alarms, both rmANOVAs showed significant main effects for group $(F(2,110)=4.4, p=.01$, $\eta \mathrm{p}^{2}=.07 ; F(2,110)=3.3, p=.04, \eta \mathrm{p}^{2}=.06$, respectively), but non-significant main effects for emotion $(H F$ 
Table 2 Emotion evaluation and face emotion recognition task baseline and change scores

\begin{tabular}{|c|c|c|c|c|c|c|}
\hline & \multicolumn{2}{|l|}{ MBCT } & \multicolumn{2}{|l|}{ TAU } & \multicolumn{2}{|l|}{ HV } \\
\hline & Baseline & Change & Baseline & Change & Baseline & Change \\
\hline Emotion evaluation & $N=40$ & & $N=33$ & & $N=42$ & \\
\hline Composite task rating & $4.1(.22)$ & $.12^{\wedge}(.28)$ & $4.1(.28)$ & $-.005(.20)$ & $4.2(.25)$ & $.01(.21)$ \\
\hline Social positive & $5.8(.51)$ & $.16(.54)$ & $5.8(.50)$ & $.02(.44)$ & $5.9(.53)$ & $-.02(.28)$ \\
\hline Social negative & $2.3(.68)$ & $.19(.43)$ & $2.2(.82)$ & $-.01(.65)$ & $2.3(.49)$ & $.06(.49)$ \\
\hline Autonomous positive & $6.4(.48)$ & $.05(.43)$ & $6.4(.49)$ & $.08(.35)$ & $6.6(.41)$ & $-.07(.24)$ \\
\hline Autonomous negative & $2.0(.52)$ & $.08(.59)$ & $2.0(.51)$ & $-.07(.45)$ & $2.2(.49)$ & $.05(.42)$ \\
\hline Neutral & $4.2(.39)$ & $.13(.45)$ & $4.3(.41)$ & $-.05(.44)$ & $4.2(.31)$ & $.05(.29)$ \\
\hline \multicolumn{7}{|l|}{ Face emotion recognition } \\
\hline Accuracy (hit proportions) & $N=38$ & & $N=33$ & & $N=42$ & \\
\hline Composite emotion recognition & $.61(.09)$ & $.04 * *(.07)$ & $.61(.07)$ & $.03(.07)$ & $.63(.09)$ & $-.0003(.05)$ \\
\hline Anger & $.32(.18)$ & $.06(.16)$ & $.34(.16)$ & $.04(.14)$ & $.33(.20)$ & $.01(.15)$ \\
\hline Disgust & $.59(.16)$ & $.06(.18)$ & $.59(.17)$ & $.07(.16)$ & $.62(.16)$ & $-.01(.12)$ \\
\hline Fear & $.53(.22)$ & $.05(.18)$ & $.51(.19)$ & $.005(.16)$ & $.52(.19)$ & $-.008(.16)$ \\
\hline Happy & $.75(.13)$ & $.08(.14)$ & $.81(.16)$ & $.02(.11)$ & $.84(.12)$ & $.02(.09)$ \\
\hline $\mathrm{Sad}$ & $.60(.17)$ & $.02(.19)$ & $.52(.20)$ & $.04(.19)$ & $.57(.18)$ & $-.03(.20)$ \\
\hline Surprise & $.70(.15)$ & $.02(.15)$ & $.66(.17)$ & $.02(.18)$ & $.68(.17)$ & $.008(.14)$ \\
\hline Neutral & $.80(.20)$ & $-.002(.15)$ & $.85(.14)$ & $.02(.11)$ & $.88(.12)$ & $.01(.15)$ \\
\hline \multicolumn{7}{|l|}{ False alarms } \\
\hline Composite false alarms & $.063(.01)$ & $-.006^{*}(.01)$ & $.064(.01)$ & $-.005(.01)$ & $.061(.01)$ & $-.0001(.01)$ \\
\hline Anger & $.047(.04)$ & $-.011(.04)$ & $.043(.04)$ & $-.010(.03)$ & $.039(.03)$ & $-.003(.03)$ \\
\hline Disgust & $.036(.03)$ & $.001(.02)$ & $.043(.03)$ & $.002(.02)$ & $.036(.03)$ & $-.006(.03)$ \\
\hline Fear & $.052(.04)$ & $-.011(.04)$ & $.052(.04)$ & $-.014(.04)$ & $.040(.03)$ & $.004(.03)$ \\
\hline Hарру & $.003(.009)$ & $.002(.01)$ & $.003(.006)$ & $.002(.01)$ & $.008(.03)$ & $.005(.02)$ \\
\hline $\mathrm{Sad}$ & $.055(.05)$ & $-.001(.04)$ & $.035(.03)$ & $-.0001(.03)$ & $.040(.04)$ & $-.0001(.04)$ \\
\hline Surprise & $.060(.03)$ & $-.005(.03)$ & $.063(.04)$ & $.005(.03)$ & $.061(.04)$ & $-.005(.04)$ \\
\hline Neutral & $.184(.08)$ & $-.001(.08)$ & $.210(.07)$ & $-.020(.06)$ & $.199(.08)$ & $.003(.05)$ \\
\hline
\end{tabular}

Values are means $(S D s)$; caret and asterisks represent Dunnett's test comparisons between MBCT group and control groups following significant ANOVA

$H V$ healthy, never depressed, volunteers

$\hat{p}<.05$ vs. TAU; $* * p<.01, * p<.05$ vs. HVs

$F(6,660)=.91, p=.49, \eta \mathrm{p}^{2}=.01 ; H F \quad F(6,660)=1.26$, $\left.p=.28, \mathrm{np}^{2}=.01\right)$, and non-significant group by emotion interactions $\left(H F F(12,660)=.81, p=.64, \eta^{2}=.01 ; H F\right.$ $\left.F(12,660)=.99, p=.44, \eta p^{2}=.02\right)$. Both rMDD groups showed positive change scores for overall accuracy (general emotion recognition), with only MBCT significantly different from HVs (Dunnett's test $p=.01$ but not TAU (Fig. 5)); both MBCT and TAU groups increased significantly over time ( $p=.002$ and $p=.01$ respectively, one-sample $t$ test). For false alarms, both rMDD groups showed negative changes scores for overall false alarms (reduction post-session); post hoc comparisons (Dunnett's test) showed a significant difference between MBCT and HVs $(p=.04)$ but not TAU (Fig. 5; Table 2); both MBCT and TAU groups decreased significantly over time ( $p=.007$ and $p=.01$ respectively, one-sample $t$ test). Sensitivity analyses showed the same pattern of results but the findings were weakened when restricted to the nonmedicated participants. The ANCOVA on the composite accuracy and false-alarm scores showed non-significant differences between the two rMDD groups (see Supplementary Material).

\section{Correlations Between Composite Change Scores and Mindfulness}

In all groups taken together, there was a significant positive correlation between the emotion evaluation task composite change score (i.e. mean score across all emotion conditions) and self-report mindfulness (FFMQ-4) change scores (Pearson $r=.28, p=.003, R^{2}=7.8 \%$ ), indicating that the 
$\mathbf{a}$
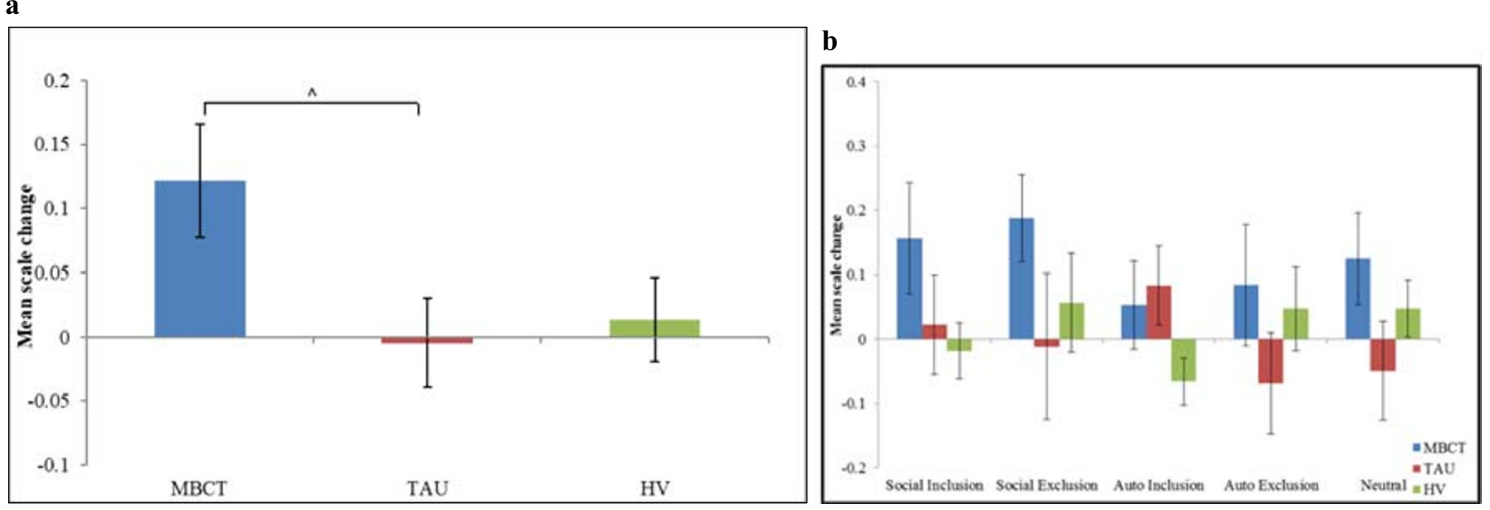

Fig. 4 a Overall emotion evaluation task change by group. Note. TAU = treatment as usual; $\mathrm{HV}=$ healthy, never depressed, volunteers; ${ }^{\wedge} p<.05$ vs. TAU. b Change scores for each condition by group (for illustration).

Note. $\mathrm{TAU}=$ treatment as usual; $\mathrm{HV}=$ healthy, never depressed, volunteers; Auto $=$ autonomous

larger the increase in self-report mindfulness, the greater the positive change in overall emotion evaluation. In the groups considered separately, there was a significant positive correlation between the emotion evaluation task composite change score and self-report mindfulness (FFMQ-4) change score in the MBCT group (Pearson $r=.41, p=.01, R^{2}=16.81 \%$ ) but no significant correlation in the TAU or HV groups, indicating that the positive relationship between self-report mindfulness and overall emotion evaluation change may be linked to changes following MBCT. Overall face emotion recognition change (accuracy or false alarms) did not correlate with selfreport mindfulness change overall or in any group.

In the MBCT group, there was a significant positive correlation between the emotion evaluation task composite change and minutes of mindfulness practice during MBCT (Pearson $\left.r=.47, p=.002, R^{2}=22.1 \%\right)$ and mean days practiced during MBCT (Pearson $r=.39, p=.01, R^{2}=15.2 \%$ ), suggesting that the more time spent practicing mindfulness, the greater the positive change in overall emotion evaluation. There were no correlations between overall face emotion recognition change and either of the mindfulness practice measures.

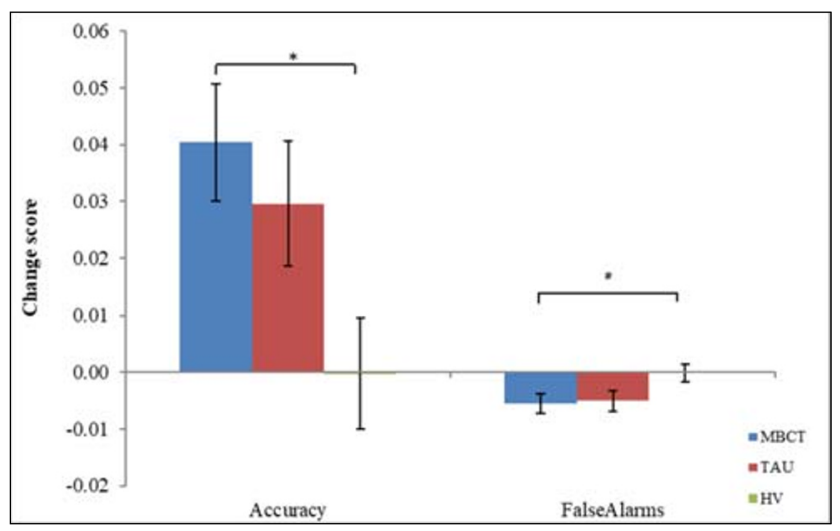

Fig. 5 Overall face emotion recognition. Note. Accuracy and false alarm change scores by group; TAU = treatment as usual; $\mathrm{HV}=$ healthy, never depressed, volunteers; $* p<.05$ vs. HVs

\section{Discussion}

In this study, we investigated change in emotional processing following MBCT in rMDD participants. In line with our hypothesis, we found significant positive changes on the emotion evaluation task following MBCT only, suggesting a shift from less negative to more positive emotion evaluations across a range of life situations. This change in the MBCT group correlated with engagement with mindfulness practice, as well as with changes in self-report mindfulness, strongly supporting that such shifts in emotion processing are specifically related to mindfulness practice. Our hypothesis was not supported with regard to face emotion recognition with no evidence that MBCT resulted in a differential effect on positive and negative emotion recognition accuracy, although it is important to take into account in this context that contrary to our expectations, there were no significant baseline differences between clinical and non-clinical groups in this task. There was an overall increase in emotion recognition accuracy compared with HVs in the MBCT group, but this was small and also occurred in the TAU group, making its interpretation unclear and unlikely to relate to MBCT.

\section{Specific Effects of MBCT}

Our key finding is that on the emotion evaluation task, rMDD participants shifted from less negative to more positive emotion evaluations following MBCT. Therefore, after 8 weeks of MBCT, participants shifted their expectation of how happy they would feel in a range of everyday scenarios. This finding seemed to be mostly driven by the social (both inclusion and exclusion) and neutral scenarios, but the lack of statistical significance between emotion conditions means that this needs to be specifically tested in further research. In support of this being a specific effect of MBCT, those who dedicated more time to practicing mindfulness in between sessions showed greater positive changes with practice duration 
explaining approximately $22 \%$ of the variance. MBCT training encourages "turning towards" all experience-pleasant, unpleasant, or neutral; thus, increases towards positive emotions across all scenarios could be a product of a more open and accepting stance towards experience developed through continued mindfulness practice during MBCT.

Our finding of a shift from less negative to more positive emotion evaluations in rMDD participants undergoing MBCT adds to the evidence from self-report studies of increases in positive emotions following MBCT. For example, other studies have reported increased self-report appraisals of positive emotions or prosocial feelings following MBCT in rMDD participants, compared with control participants (Batink et al. 2013; Collip et al. 2013; Garland et al. 2015; Geschwind et al. 2011). Further studies have reported similar increases in positive affect across different MBIs in clinical populations. For example, Garland et al. (2017) reported increases in positive affect in a sample of participants with social anxiety disorder (SAD) undergoing MBSR, compared with those undergoing CBT. Further, in an uncontrolled study, Strege et al. (2018) reported that increases in positive affect predicted social anxiety symptom reduction in participants undergoing MBCT. Our finding adds evidence for shifts towards positive emotion evaluations in experimental, contextualised scenarios.

Other studies have shown that mindfulness training improves positivity ratings of life events, promotes social behaviour, and reduces social phobia and loneliness (Beauchemin et al. 2008; Creswell et al. 2012; Nezlek et al. 2016; Piet et al. 2010). Increasing positive emotions in social scenarios may be particularly relevant for individuals either in remission or currently depressed who may be increasingly isolating or withdrawing from social situations (Cruwys et al. 2014). Given that the inability to experience pleasure is a core symptom of the experience of and a diagnosis of major depression (Pizzagalli et al. 2008) and persists to some degree into remission (Pechtel et al. 2013), our finding suggests that MBCT may be modifying an important vulnerability which contributes to the maintenance of depression.

\section{Non-specific Effects of MBCT}

However, on the face emotion recognition task, we identified changes in both of the MBCT and TAU groups, but not in the HVs, although this was not related to specific emotions. This is similar to findings by De Raedt et al. (2012), who found reduced inhibition towards positive faces following MBCT and TAU, and might point towards a non-specific effect of MBCT on face emotion recognition. This result is unlikely to be due to task familiarity, practice, or chance as there were no changes over time in HVs. The wider evidence base for whether face emotion recognition deficits seen in major depression persist into remission is unclear (Anderson et al. 2011; Bouhuys et al. 1999; Joorman \& Gotlib, 2007) and our rMDD sample, which had depression scores within a mild to normal range, did not significantly differ from $\mathrm{HVs}$ on the face emotion recognition task at baseline. While the changes in face recognition we observed could point towards a general trend towards improvements in the processing of facial stimuli, the lack of a baseline deficit makes interpretation of this small change unclear.

\section{Implications}

Our study suggests that MBCT encourages a shift towards more positive emotion evaluations across a range of scenarios in previously depressed participants. Importantly, our study was able to show this using a more experimental measure giving complementary evidence to that obtained from self-report questionnaires which have intrinsic limitations, for example, response-demand expectation. This is consistent with mindfulness practices leading to more "eudaimonic" processing (Garland et al. 2015), i.e. taking a more positive approach to life in general, encompassing positive, negative, and neutral aspects of our experience (Ryan and Deci 2000). Given that a reduced ability to experience pleasure is one of two core symptoms in the diagnosis of major depression and that a blunted reward response may persist into remission (Pechtel et al. 2013), our study suggests an important mechanism by which MBCT affects emotional processing for people in remission from depression, and is potentially involved in protecting against depressive relapse.

Our finding provides experimental support consistent with the broad aim of MBCT to encourage a more open, less judgemental, broadening of awareness to embrace all emotional experience, and suggests that mindfulness training can indeed help to encourage a move towards embracing more positive aspects of experience. This could contribute to countering negative biases that make people vulnerable to relapse into depression.

\section{Limitations and Future Research}

Our study was relatively small, with under-recruitment to the TAU group, and was only powered to detect a moderate-tolarge effect so smaller group differences may have been missed, especially those related to specific emotional conditions. Another important limitation of our study is that the rMDD groups were not randomised and participants chose whether they wanted to participate in MBCT or TAU. In spite of the finding being supported by the sensitivity analysis using ANCOVA to control for any baseline differences, we cannot exclude changes in the MBCT group being attributable to underlying expectations of improvement, motivation, or belief in MBCT. Further, potential bias was also induced by including early MBCT dropouts in the TAU group but sensitivity analyses argue strongly against this, with the ITT population showing the same results including in 
the ANCOVA of rMDD participants. We cannot comment on the internal reliability of our tasks which is not straightforward to analyse or interpret given that individual task conditions may measure different aspects or constructs. The ICC results do however provide some reassurance around task test-retest reliability which has been argued to be more important in determining test stability and utility than internal reliability (McCrae et al. 2011).

Further, the positive correlations we found between changes in emotion evaluation, MBCT practice, and self-report ratings we believe argue against our finding being due to these potential confounds and support the changes we found being related mechanistically to MBCT. Although the magnitude of the positive change was relatively small, it is consistent with the difference found between depressed participants and healthy volunteers in a preliminary study (Lythe 2006). This might be explained by the task being of a non-personalised and non-interactive nature although we would argue that it allows for standardised application. Finally, although we did not find a significant improvement in mood following MBCT, as measured by the MADRS, it is possible that our findings reflect a more subtle background change in positive and negative emotions outside of the experimental situation, and this could be incorporated into future research. Our results should be reproduced in larger, randomised controlled trials and extended to investigate the relationship between changes in emotional processing and clinical outcomes such as depressive relapse. Further development of experimental paradigms could consider a more self-referential approach to make the context more individualised for participants.

Acknowledgements The authors are very grateful to Kelly Birtwell who co-facilitated all of the MBCT groups and to Merryn Dowson for her assistance with data collection.

Author Contributions KW designed and executed the study, analysed the data, and drafted and edited the manuscript. RE, RZ, TB, and IA supervised the design, study completion, and the analysis, and all authors contributed to the drafting and editing of the manuscript. TB provided clinical supervision for all of the MBCT groups. All authors have approved the final version of the manuscript for submission.

Funding This work was completed as part of the lead author's $\mathrm{PhD}$ studies with funding from both the EPSRC (Engineering and Physical Sciences Research Council) and an internal funding award from The University of Manchester.

\section{Compliance with Ethical Standards}

The authors declare that they have no conflict of interest.

Ethical Approval was granted by the NHS (North West - Preston Research Ethics Committee). All participants gave written informed consent in line with the Declaration of Helsinki (World Medical Association 2001).
Open Access This article is licensed under a Creative Commons Attribution 4.0 International License, which permits use, sharing, adaptation, distribution and reproduction in any medium or format, as long as you give appropriate credit to the original author(s) and the source, provide a link to the Creative Commons licence, and indicate if changes were made. The images or other third party material in this article are included in the article's Creative Commons licence, unless indicated otherwise in a credit line to the material. If material is not included in the article's Creative Commons licence and your intended use is not permitted by statutory regulation or exceeds the permitted use, you will need to obtain permission directly from the copyright holder. To view a copy of this licence, visit http://creativecommons.org/licenses/by/4.0/.

\section{References}

Anderson, I. M., Shippen, C., Juhasz, G., Chase, D., Thomas, E., Downey, D., Toth, Z. G., Lloyd-Williams, K., Elliott, R., \& Deakin, J. F. W. (2011). State-dependent alteration in face emotion recognition in depression. British Journal of Psychiatry, 198(4), 302-308. https://doi.org/10.1192/bjp.bp.110.078139.

Baer, R. A., Smith, G. T., Hopkins, J., Krietemeyer, J., \& Toney, L. (2006). Using self-report assessment methods to explore facets of mindfulness. Assessment, 13(1), 27-45. https://doi.org/10.1177/ 1073191105283504.

Batink, T., Peeters, F., Geschwind, N., van Os, J., \& Wichers, M. (2013). How does MBCT for depression work? Studying cognitive and affective mediation pathways. PLoS One, 8(8), 1-13. https://doi. org/10.1371/journal.pone.0072778.

Beauchemin, J., Hutchins, T. L., \& Patterson, F. (2008). Mindfulness meditation may lessen anxiety, promote social skills, and improve academic performance among adolescents with learning disabilities. Complementary Health Practice Review, 13(1), 34-45. https://doi. org/10.1177/1533210107311624.

Beck, A. T. (1967). Depression: clinical, experimental, and theoretical aspects. Harper \& Row.

Beck, A. T. (2008). The evolution of the cognitive model of depression and its neurobiological correlates. American Journal of Psychiatry, 165(969-977). https://doi.org/10.1176/appi.ajp.2008.08050721.

Bouhuys, A. L., Geerts, E., \& Gordijn, M. C. (1999). Depressed patients' perceptions of facial emotions in depressed and remitted states are associated with relapse: a longitudinal study. The Journal of Nervous and Mental Disease, 187(10), 595-602 http://www.ncbi. nlm.nih.gov/pubmed/10535652.

Bourke, C., Douglas, K., \& Porter, R. (2010). Processing of facial emotion expression in major depression: a review. Australian \& New Zealand Journal of Psychiatry, 44(8), 681-696. https://doi.org/10. 3109/00048674.2010.496359

Collip, D., Geschwind, N., Peeters, F., Myin-Germeys, I., van Os, J., \& Wichers, M. (2013). Putting a hold on the downward spiral of paranoia in the social world: a randomized controlled trial of mindfulness-based cognitive therapy in individuals with a history of depression. PLoS One, 8(6), e66747. https://doi.org/10.1371/ journal.pone.0066747.

Creswell, J. D., Irwin, M. R., Burklund, L. J., Lieberman, M. D., Arevalo, J. M. G., Ma, J., Breen, E. C., \& Cole, S. W. (2012). Mindfulnessbased stress reduction training reduces loneliness and proinflammatory gene expression in older adults: a small randomized controlled trial. Brain, Behavior, and Immunity, 26(7), 1095-1101. https://doi.org/10.1016/j.bbi.2012.07.006.

Cruwys, T., Alexander Haslam, S., Dingle, G. A., Jetten, J., Hornsey, M. J., Desdemona Chong, E. M., \& Oei, T. P. S. (2014). Feeling connected again: interventions that increase social identification reduce 
depression symptoms in community and clinical settings. Journal of Affective Disorders, 159, 139-146. https://doi.org/10.1016/j.jad. 2014.02.019.

De Raedt, R., Baert, S., Demeyer, I., Goeleven, E., Raes, A., Visser, A., Wysmans, M., Jansen, E., Schacht, R., Van Aalderen, J. R., \& Speckens, A. (2012). Changes in attentional processing of emotional information following mindfulness-based cognitive therapy in people with a history of depression: towards an open attention for all emotional experiences. Cognitive Therapy and Research, 36(6), 612-620. https://doi.org/10.1007/s10608-011-9411-x.

Disner, S. G., Beevers, C. G., Haigh, E. A. P., \& Beck, A. T. (2011). Neural mechanisms of the cognitive model of depression. Nature Reviews Neuroscience, 12, 467-477. https://doi.org/10.1038/ nrn3027.

Ekman, P. (1992). An argument for basic emotions. Cognition \& Emotion, 6(3), 169-200. https://doi.org/10.1080/02699939208411068.

Ekman, P., \& Friesen, W. (1976). Pictures of facial affect. Palo Alto: Consulting Psychologists Press.

Elliott, R., Lythe, K., Lee, R., McKie, S., Juhasz, G., Thomas, E. J., Downey, D., Deakin, J. F. W., \& Anderson, I. M. (2012). Reduced medial prefrontal responses to social interaction images in remitted depression. Archives of General Psychiatry, 69(1), 3745. https://doi.org/10.1001/archgenpsychiatry.2011.139.

Garland, E. L., Fredrickson, B., Kring, A. M., Johnson, D. P., Meyer, P. S., \& Penn, D. L. (2010). Upward spirals of positive emotions counter downward spirals of negativity: insights from the broaden-andbuild theory and affective neuroscience on the treatment of emotion dysfunctions and deficits in psychopathology. Clinical Psychology Review, 30(7), 849-864. https://doi.org/10.1016/j.cpr.2010.03.002.

Garland, E. L., Geschwind, N., Peeters, F., \& Wichers, M. (2015). Mindfulness training promotes upward spirals of positive affect and cognition: multilevel and autoregressive latent trajectory modeling analyses. Frontiers in Psychology, 6(1-13), 15. https://doi.org/ 10.3389/fpsyg.2015.00015.

Garland, E. L., Hanley, A. W., Goldin, P. R., \& Gross, J. J. (2017). Testing the mindfulness-to-meaning theory: evidence for mindful positive emotion regulation from a reanalysis of longitudinal data. PLoS One, 12(12), e0187727. https://doi.org/10.1371/journal.pone.0187727.

Geschwind, N., Peeters, F., Drukker, M., van Os, J., \& Wichers, M. (2011). Mindfulness training increases momentary positive emotions and reward experience in adults vulnerable to depression: a randomized controlled trial. Journal of Consulting and Clinical Psychology, 79(5), 618-628. https://doi.org/10.1037/a0024595.

Gu, J., Strauss, C., Bond, R., \& Cavanagh, K. (2015). How do mindfulness-based cognitive therapy and mindfulness-based stress reduction improve mental health and wellbeing? A systematic review and meta-analysis of mediation studies. Clinical Psychology Review, 37, 1-12. https://doi.org/10.1016/j.cpr.2015.01.006.

Hargus, E., Crane, C., Barnhofer, T., \& Williams, J. M. G. (2010). Effects of mindfulness on meta-awareness and specificity of describing prodromal symptoms in suicidal depression. Emotion, 10(1), 34-42. https://doi.org/10.1037/a0016825.

Henriques, J. B., \& Davidson, R. J. (2000). Decreased responsiveness to reward in depression. Cognition \& Emotion, 14(5), 711-724. https:// doi.org/10.1080/02699930050117684.

Joormann, J., \& Gotlib, I. H. (2007). Selective attention to emotional faces following recovery from depression. Journal of Abnormal Psychology, 116(1), 80-85. https://doi.org/10.1037/0021-843X.116.1.80.

Kupferberg, A., Bicks, L., \& Hasler, G. (2016). Social functioning in major depressive disorder. Neuroscience \& Biobehavioral Reviews, 69, 313-332. https://doi.org/10.1016/J.NEUBIOREV.2016.07.002.

LeMoult, J., Joormann, J., Sherdell, L., Wright, Y., \& Gotlib, I. H. (2009). Identification of emotional facial expressions following recovery from depression. Journal of Abnormal Psychology, 118(4), 828833. https://doi.org/10.1037/a0016944.
Leppänen, J. M. (2006). Emotional information processing in mood disorders: a review of behavioral and neuroimaging findings. Current Opinion in Psychiatry, 19(1), 34-39. https://doi.org/10.1097/01. yco.0000191500.46411.00.

Lythe, K., (2006). The role of monoamines in mood, reinforcement and impulsivity. (PhD Thesis). The University of Manchester, Manchester.

McCabe, S. B., \& Gotlib, I. H. (1995). Selective attention and clinical depression: performance on a deployment-of-attention task. Journal of Abnormal Psychology, 104(1), 241-245. https://doi.org/10.1037/ 0021-843X.104.1.241.

McCrae, R. R., Kurtz, J. E., Yamagata, S., \& Terracciano, A. (2011) Internal consistency, retest reliability, and their implications for personality scale validity. Personality and Social Psychology Review, 15(1), 28-50. https://doi.org/10.1177/1088868310366253.

Michalak, J., Hölz, A., \& Teismann, T. (2011). Rumination as a predictor of relapse in mindfulness-based cognitive therapy for depression. Psychology and Psychotherapy: Theory, Research and Practice, 84(2), 230-236. https://doi.org/10.1348/147608310X520166.

Montgomery, S. A., \& Asberg, M. (1979). A new depression scale designed to be sensitive to change. British Journal of Psychiatry, 134(9), 382-389.

Münkler, P., Rothkirch, M., Dalati, Y., Schmack, K., \& Sterzer, P. (2015). Biased recognition of facial affect in patients with major depressive disorder reflects clinical state. PLoS One, 10(6), e0129863. https://doi.org/10.1371/journal.pone.0129863.

Nezlek, J. B., Holas, P., Rusanowska, M., \& Krejtz, I. (2016). Being present in the moment: event-level relationships between mindfulness and stress, positivity, and importance. Personality and Individual Differences, 93, 1-5. https://doi.org/10.1016/j.paid. 2015.11.031.

Pechtel, P., Dutra, S. J., Goetz, E. L., \& Pizzagalli, D. A. (2013). Blunted reward responsiveness in remitted depression. Journal of Psychiatric Research, 47(12), 1864-1869. https://doi.org/10.1016/ J.JPSYCHIRES.2013.08.011.

Piet, J., Hougaard, E., Hecksher, M. S., \& Rosenberg, N. K. (2010). A randomized pilot study of mindfulness-based cognitive therapy and group cognitive-behavioral therapy for young adults with social phobia. Scandinavian Journal of Psychology, 51(5), 403-410. https://doi.org/10.1111/j.1467-9450.2009.00801.x.

Pizzagalli, D. A., Jahn, A. L., \& O'Shea, J. P. (2005). Toward an objective characterization of an anhedonic phenotype: a signal-detection approach. Biological Psychiatry, 57(4), 319-327. https://doi.org/10. 1016/J.BIOPSYCH.2004.11.026.

Pizzagalli, D. A., Iosifescu, D., Hallett, L. A., Ratner, K. G., \& Fava, M. (2008). Reduced hedonic capacity in major depressive disorder: evidence from a probabilistic reward task. Journal of Psychiatric Research, 43(1), 76-87. https://doi.org/10.1016/j.jpsychires.2008. 03.001.

Psychological Corporation. (2001). The Wechsler Test of Adult Reading (WTAR):test manual. The Psychological Corporation.

Psychology Software Tools (2012). Computer software E-Prime 2.0. Psychology Software Tools.

Rhebergen, D., Beekman, A. T. F., de Graaf, R., Nolen, W. A., Spijker, J., Hoogendijk, W. J., \& Penninx, B. W. J. H. (2010). Trajectories of recovery of social and physical functioning in major depression, dysthymic disorder and double depression: a 3-year follow-up. Journal of Affective Disorders, 124(1-2), 148-156. https://doi.org/ 10.1016/j.jad.2009.10.029.

Ryan, R. M., \& Deci, E. L. (2000). Self-determination theory and the facilitation of intrinsic motivation, social development, and wellbeing. The American Psychologist, 55(1), 68-78 http://www.ncbi. nlm.nih.gov/pubmed/11392867.

Saris, I. M. J., Aghajani, M., van der Werff, S. J. A., van der Wee, N. J. A. \& Penninx, B. W. J. H. (2017). Social functioning in patients with 
depressive and anxiety disorders. Acta Psychiatrica Scandinavica, 136(4), 352-361. https://doi.org/10.1111/acps.12774.

Scher, C. D., Ingram, R. E., \& Segal, Z. V. (2005). Cognitive reactivity and vulnerability: empirical evaluation of construct activation and cognitive diatheses in unipolar depression. Clinical Psychology Review, 25(4), 487-510. https://doi.org/10.1016/j.cpr.2005.01.005.

Segal, Z. V., Kennedy, S., Gemar, M., Hood, K., Pedersen, R., \& Buis, T. (2006). Cognitive reactivity to sad mood provocation and the prediction of depressive relapse. Archives of General Psychiatry, 63(7), 749. https://doi.org/10.1001/archpsyc.63.7.749-755.

Segal, Z. V., Williams, J. M. G., \& Teasdale, J. D. (2013). Mindfulnessbased cognitive therapy for depression. The Guildford Press.

Shahar, B., Britton, W. B., Sbarra, D. A., Figueredo, A. J., \& Bootzin, R. R. (2010). Mechanisms of change in mindfulness-based cognitive therapy for depression: preliminary evidence from a randomized controlled trial. International Journal of Cognitive Therapy, 3(4), 402-418. https://doi.org/10.1521/ijct.2010.3.4.402.

Sheehan, D. V., Lecrubier, Y., Sheehan, K. H., Amorim, P., Janavs, J., Weiller, E., Hergueta, T., Baker, R., \& Dunbar, G. C. (1998). The Mini-International Neuropsychiatric Interview (M.I.N.I.): the development and validation of a structured diagnostic psychiatric interview for DSM-IV and ICD-10. The Journal of Clinical Psychiatry, 59 Suppl 20(22-33), 34-57. http://www.ncbi.nlm.nih.gov/pubmed/ 9881538.

Shiroma, P. R., Thuras, P., Johns, B., \& Lim, K. O. (2016). Facial recognition of happiness among older adults with active and remitted major depression. Psychiatry Research, 243, 287-291. https://doi. org/10.1016/j.psychres.2016.06.020.

Strege, M. V., Swain, D., Bochicchio, L., Valdespino, A., \& Richey, J. A. (2018). A pilot study of the effects of mindfulness-based cognitive therapy on positive affect and social anxiety symptoms. Frontiers in Psychology, 9, 866 (1-13). https://doi.org/10.3389/fpsyg.2018. 00866.

Teasdale, J. D., Moore, R. G., Hayhurst, H., Pope, M., \& Williams, S. (2002). Metacognitive awareness and prevention of relapse in depression: empirical evidence. Journal of Consulting and Clinical Psychology, 70(2), 275-287. https://doi.org/10.1037//0022-006X. 70.2.275.

van Aalderen, J. R., Donders, A. R. T., Giommi, F., Spinhoven, P., Barendregt, H. P., \& Speckens, A. E. M. (2012). The efficacy of mindfulness-based cognitive therapy in recurrent depressed patients with and without a current depressive episode: a randomized controlled trial. Psychological Medicine, 42(05), 989-1001. https://doi. org/10.1017/S0033291711002054.

van Breukelen, G. J. P. (2013). ANCOVA versus change from baseline in nonrandomized studies: the difference. Multivariate Behavioral Research, 48(6), 895-922. https://doi.org/10.1080/00273171.2013. 831743.

van Den Hurk, P. A. M., van Aalderen, J. R., Giommi, F., Donders, R. A. R. T., Barendregt, H. P., \& Speckens, A. E. M. (2012). An investigation of the role of attention in mindfulness-based cognitive therapy for recurrently depressed patients. Journal of Experimental Psychopathology JEP, 3(1), 103-120. https://doi.org/10.5127/jep. 024811.

Weightman, M. J., Air, T. M., \& Baune, B. T. (2014). A review of the role of social cognition in major depressive disorder. Frontiers in Psychiatry, 5, article 179 (1-13). https://doi.org/10.3389/fpsyt. 2014.00179 .

Weinberg, A., \& Shankman, S. A. (2017). Blunted reward processing in remitted melancholic depression. Clinical Psychological Science, 5(1), 14-25. https://doi.org/10.1177/2167702616633158.

Williams, K. (2018). Neuropsychological mechanisms of mindfulnessbased cognitive therapy for depression. (PhD Thesis). The University of Manchester, Manchester. https://ethos.bl.uk/ OrderDetails.do?uin=uk.bl.ethos.748014.

Williams, M. J., Dalgleish, T., Karl, A., \& Kuyken, W. (2014). Examining the factor structures of the Five Facet Mindfulness Questionnaire and the Self-Compassion Scale. Psychological Assessment, 26(2), 407-418. https://doi.org/10.1037/a0035566.

Williams, K., Elliott, R., McKie, S., Zahn, R., Barnhofer, T., \& Anderson, I. M. (2020). Changes in the neural correlates of self-blame following mindfulness-based cognitive therapy in remitted depressed participants. Psychiatry Research - Neuroimaging, 304, 111152. https://doi.org/10.1016/j.pscychresns.2020.111152.

World Medical Association. (2001). World Medical Association declaration of Helsinki. Ethical principles for medical research involving human subjects. Bulletin of the World Health Organization, 79(4), 373-374. https://apps.who.int/iris/handle/10665/268312.

Publisher's Note Springer Nature remains neutral with regard to jurisdictional claims in published maps and institutional affiliations. 